\title{
Measurement of Deformations by MEMS Arrays, Verified at Sub-millimetre Level Using Robotic Total Stations
}

\author{
Tomas Beran ${ }^{1}$, Lee Danisch ${ }^{1}$, Adam Chrzanowski ${ }^{2}$, Maciej Bazanowski ${ }^{2}$ \\ ${ }^{1}$ Measurand Inc., 2111 Hanwell Road, Fredericton, New Brunswick, E3C 1M7 Canada, Web E- \\ mail: tomas@measurand.com, lee@measurand.com \\ ${ }^{2}$ Canadian Centre for Geodetic Engineering, University of New Brunswick, PO Box 4400, \\ Fredericton, New Brunswick, E3B 5A3 Canada, E-mail: adamc@unb.ca, \\ maciej.bazanowski@unb.ca
}

\begin{abstract}
Measurement of sub-millimetre-level deformations of structures in the presence of ambient temperature changes can be challenging. This paper describes the measurement of a structure moving due to temperature changes, using two ShapeAccelArray (SAA) instruments, and verified by a geodetic monitoring system. SAA is a geotechnical instrument often used for monitoring of displacements in soil. SAA uses micro-electromechanical system (MEMS) sensors to measure tilt in the gravity field. The geodetic monitoring system, which uses ALERT software, senses the displacements of targets relative to control points, using a robotic total station (RTS). The test setup consists of a central four-metre free-standing steel tube with other steel tubes welded to most of its length. The central tube is anchored in a concrete foundation. This composite "pole" is equipped with two SAAs as well as three geodetic prisms mounted on the top, in the middle, and in the foundation. The geodetic system uses multiple control targets mounted in concrete foundations of nearby buildings, and at the base of the pole. Long-term observations using two SAAs indicate that the pole is subject to deformations due to cyclical ambient temperature variations causing the pole to move by a few millimetres each day. In a multiple-day experiment, it was possible to track this movement using SAA as well as the RTS system. This paper presents data comparing the measurements of the two instruments and provides a good example of the detection of two-dimensional movements of seemingly rigid objects due to temperature changes.
\end{abstract}

Key words: deformation monitoring, geodetic systems, geotechnical instrumentation

\section{Introduction}

Automated geodetic and geotechnical monitoring systems are playing a rapidly-increasing role in risk-reduction efforts concerning structures and their interaction with soils. Geodetic monitoring systems originated in the measurements and representation of the surface of the Earth, while the geotechnical systems originated in the study of behaviour of earth materials ([5]). Geodetic monitoring systems are represented in this paper by an automated deformation monitoring system developed by the Canadian Centre for Geodetic Engineering at the University of New Brunswick. The system in this example relies on measurements of angles and distances by a robotic total station (RTS), relative to stable reference points. ALERT uses proprietary software ([1]) for automated data collection, data transfer and data processing.

Geotechnical monitoring systems are represented here by a shape sensing instrument called ShapeAccelArray (SAA). SAA is an array of rigid segments connected by flexible joints that can 
bend in any direction, but cannot twist. Each segment is equipped with three MEMS accelerometers, sensing three orthogonal components of gravity ([3]). In a typical near vertical installation, the 3D shape of the static SAA is determined by sensing the acceleration of the $\mathrm{X}$ and $\mathrm{Y}$ accelerometers, knowledge of the segment length $(50 \mathrm{~cm}$ in this case) and by employing rotational transforms relating one segment to the next segment ([2]). The accelerometer output is influenced by the temperature, so all SAAs include digital temperature sensors, used to compensate the MEMS sensors for temperature-induced errors.

Objectives of the experiment included verifying the self-consistency of SAA measurements (by comparing the two SAA results), and verifying the precision of SAAs by comparing SAA results to those from the RTS system, used as a de facto standard. Of particular interest was the verification of new temperature compensation algorithms in the SAA software, in a field setting.

The test setup described in this paper is located in Hanwell, New Brunswick, Canada. It consists of two 4-metre vertical SAAs (with eight $50 \mathrm{~cm}$ long segments) inserted in $2.5 \mathrm{~cm}$ diameter steel pipes. There are four $2.5 \mathrm{~cm}$ diameter pipes and one $5 \mathrm{~cm}$ diameter steel pipe mounted on the circumference of a $12.5 \mathrm{~cm}$ diameter steel pipe. This 4 metre-high vertical assembly is referred to as the "pole" in this paper. The $12.5 \mathrm{~cm}$ diameter steel pipe is anchored in a 1.2 metre-deep concrete foundation. The geodetic network surrounding the pole consists of four control points located in the concrete foundations of nearby buildings (Control1, Cotrol2, Control3, Control4), one control point located in the concrete foundation of the pole (Control5), two observed points located at the top and in the middle of the pole (TOP, MIDDLE), and one RTS located about $5 \mathrm{~m}$ East of the pole. The azimuth between points Station-Controll was selected as $0^{\circ} 0^{\prime} 0^{\prime \prime}$ and roughly coincides with magnetic north. Figure 1 shows the layout of the monitoring network and the location of control points on the pole. Figure 2 shows the location of control points Control 1 , Control 2 and Control3 in the foundation of a nearby building.

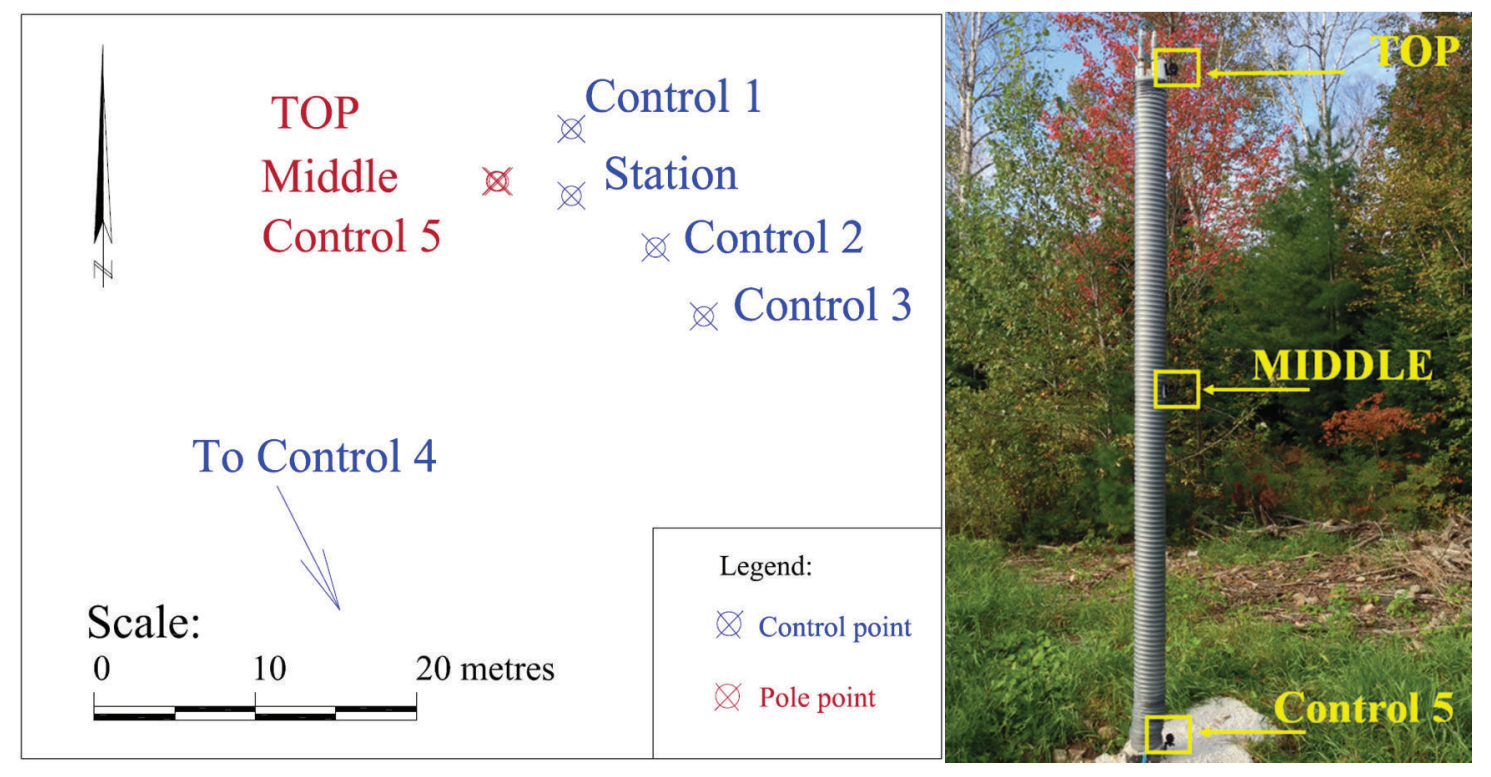

Figure 1: Layout of the geodetic network surrounding the pole holding SAAs and location of observed points on the pole.

The data for this experiment were collected on September 18-20, 2013. The RTS used in this case was a Leica TCA 1800. All control points were equipped with Leica "mini prisms". RTS measurements were automatically collected and processed using the ALERT software suite. The data collected by the two SAAs for about two months prior to the experiment indicate that there is about $2 \mathrm{~mm}$ movement observed on the top of the pole, in mostly the North-South direction. This knowledge led to the selection of a RTS with a one arc second angular resolution. Placing 
this instrument about $5 \mathrm{~m}$ from the pole, perpendicular to the direction of the movement, should result in $0.025 \mathrm{~mm}$ resolution in the movement of the pole. The size of the movement depends largely on the temperature variation, so for that reason the measurements took place on the three consecutive days with a large temperature variation. Figure 3 shows maximum daily temperature of $30{ }^{\circ} \mathrm{C}$ on day $1,33{ }^{\circ} \mathrm{C}$ on day 2 , and $29^{\circ} \mathrm{C}$ on day 3 .

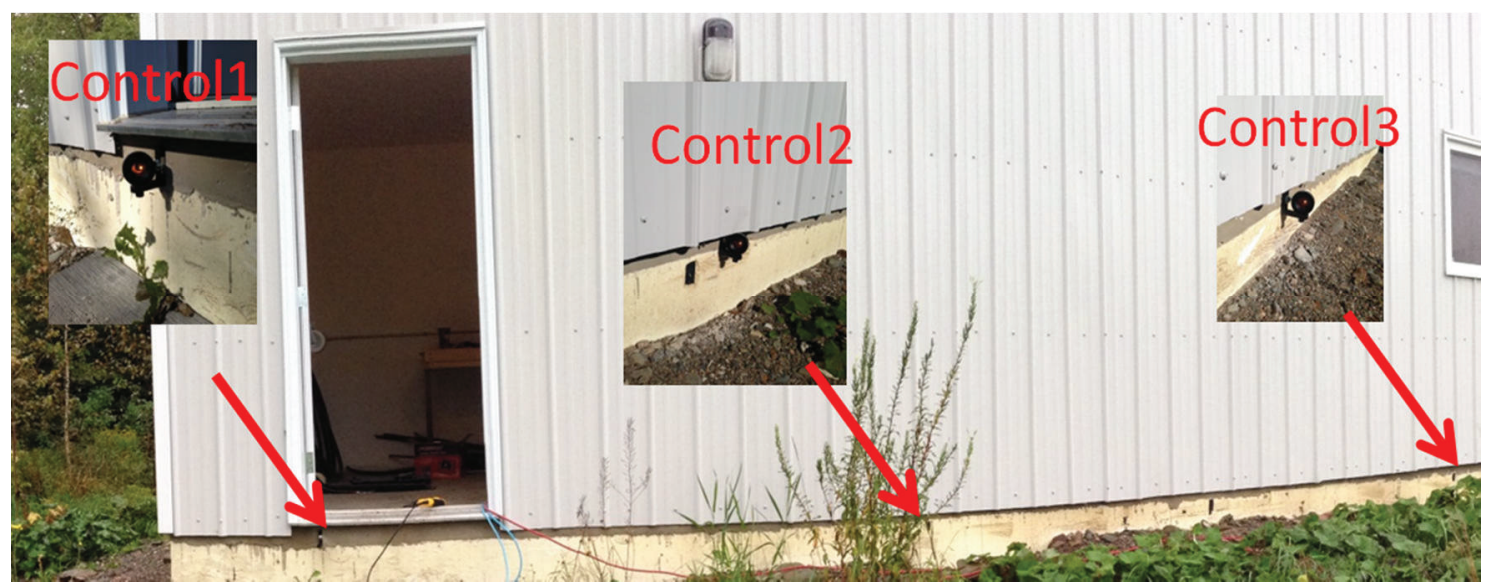

Figure 2: Location of control points Control1, Control2, and Control3.

Minimum daily temperatures of about $8{ }^{\circ} \mathrm{C}$ were observed during the first night and about $13{ }^{\circ} \mathrm{C}$ during the second night. "Hour 0" corresponds with 11:00 am local time September 18, 2013. There are data gaps from Hour 15 till Hour 22 and from Hour 38 to Hour 46 which was caused by RTS power interruptions. The temperature sensors are located in the fourth segment of the SAA relative to the cable (top) end, with a sensor resolution of $0.06{ }^{\circ} \mathrm{C}$. The precision of the $4 \mathrm{~m}$ long SAA is specified to be $0.53 \mathrm{~mm}$ ([4]).

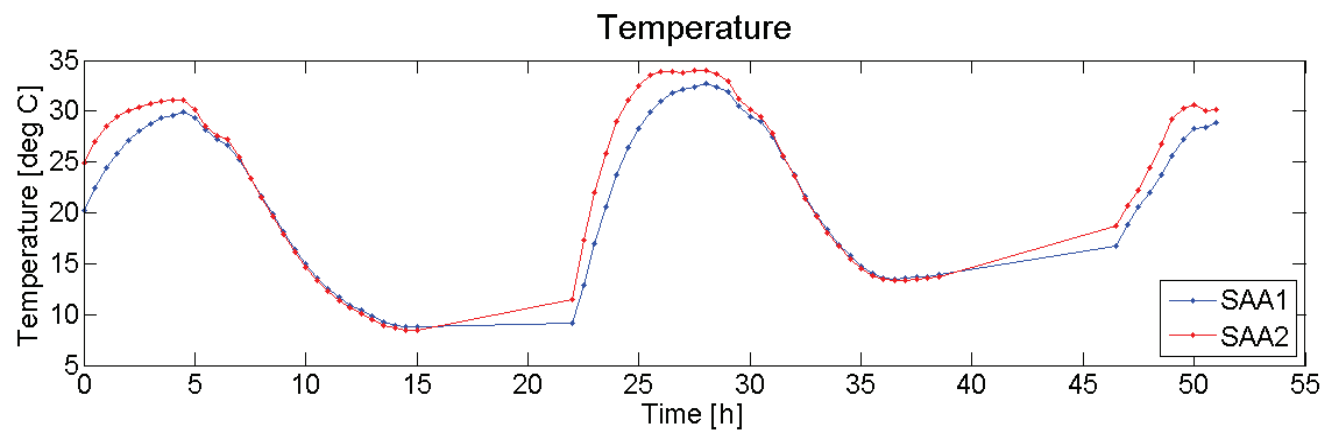

Figure 3: Temperature variation during the experiment.

\section{Analysis}

The RTS data collection cycle (3 sets of angles) took about 20 minutes to complete which led to the displacement calculation interval of 30 minutes. The data collection interval for the SAAs was about 10 minutes and the data were down-sampled to 30 minutes to match the RTS displacement calculation interval. The reference epoch for RTS and SAA data collection was set to Hour 0 and calculated deformations for this epoch are $\mathrm{X}=0 \mathrm{~mm}$ and $\mathrm{Y}=0 \mathrm{~mm}$. The $\mathrm{X}$-axes of the two SAAs were manually oriented in the direction of the nearby buildings, so it was necessary to match the orientation of the X-axis of SAA1 and SAA2 and RTS North. This was accomplished by mathematically rotating the SAA1 and SAA2 displacements around the gravity vector. The path travelled by the TOP point during the observation period according to SAA1 and RTS is shown 
in Figure 4 and the path travelled by the TOP point during the same period according to SAA2 and RTS is shown in Figure 5.

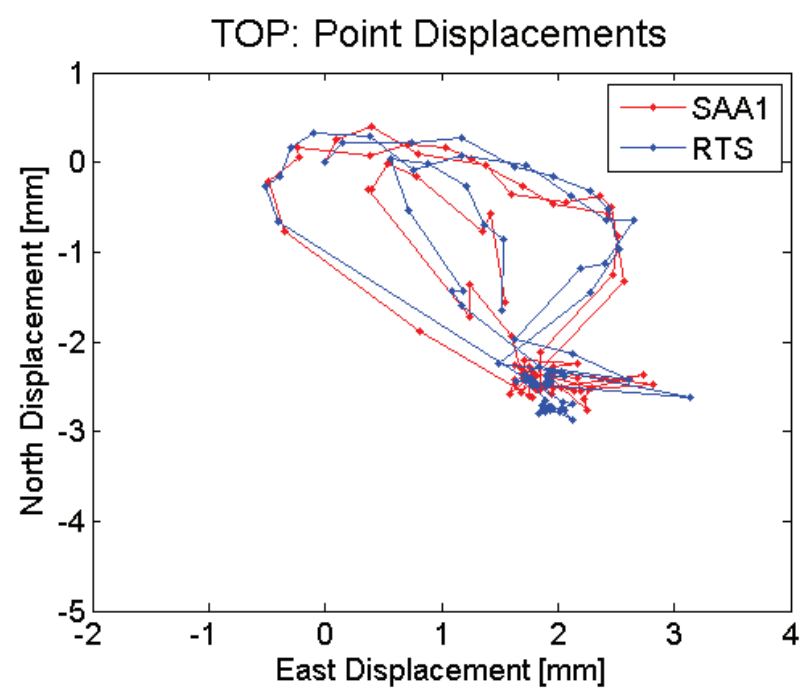

Figure 4: The path travelled by the TOP point during the observation period measured by the SAA1 (red) and RTS (blue).

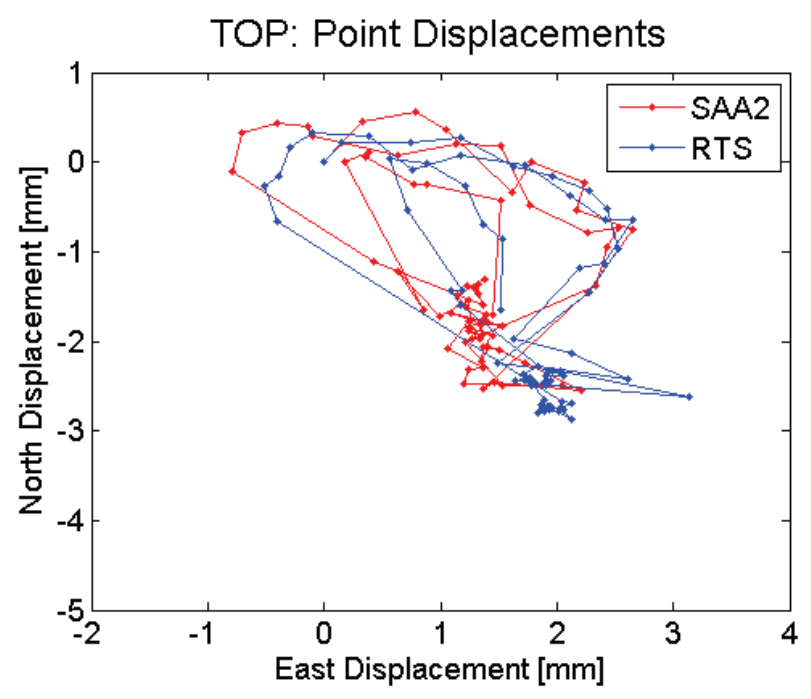

Figure 5: The path travelled by the TOP point during the observation period measured by the SAA2(red) and RTS (blue).

Figure 4 and Figure 5 show TOP point movement from the Northwest point to the Northeast point in about 4 hours. After that the TOP point moves Southwest for about 2 hours and stays there for the next 9 hours. After that there is a data gap from about Hour 15 to Hour 22 when the TOP of the pole returns Northwest and the cycle repeats in the next 24 hours.

The resulting East and North deformations for the TOP point for SAA1 and RTS are shown in Figure 6 and for SAA2 and RTS are shown in Figure 7. Both SAA1 and SAA2 and RTS instrument on both sets of plots show changes in displacements during periods of the temperature rise or Hour 0 to Hour 5 and about 24 hours later (Hour 24 to Hour 29) (see Figure 3). The spikes measured by both types of instruments at Hour 5 to 6 and Hour 29 to 30 in the East Displacement, are thought to reflect a discontinuity in the temperature-driven deformation of the pole, perhaps 
due to the welds between the multiple pipes of the pole. Other characteristics will be discussed after Figure 9.
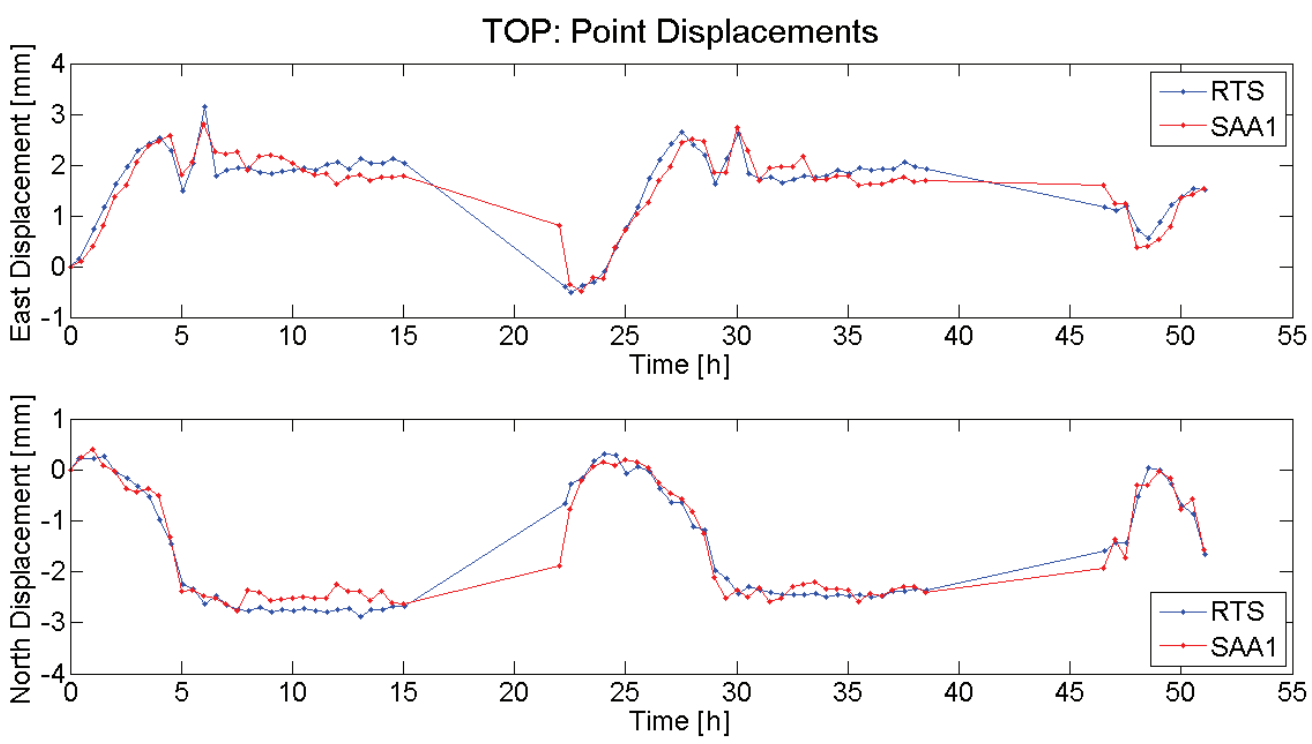

Figure 6: East and North displacements for SAA1 (red) and RTS (blue) for the TOP point.
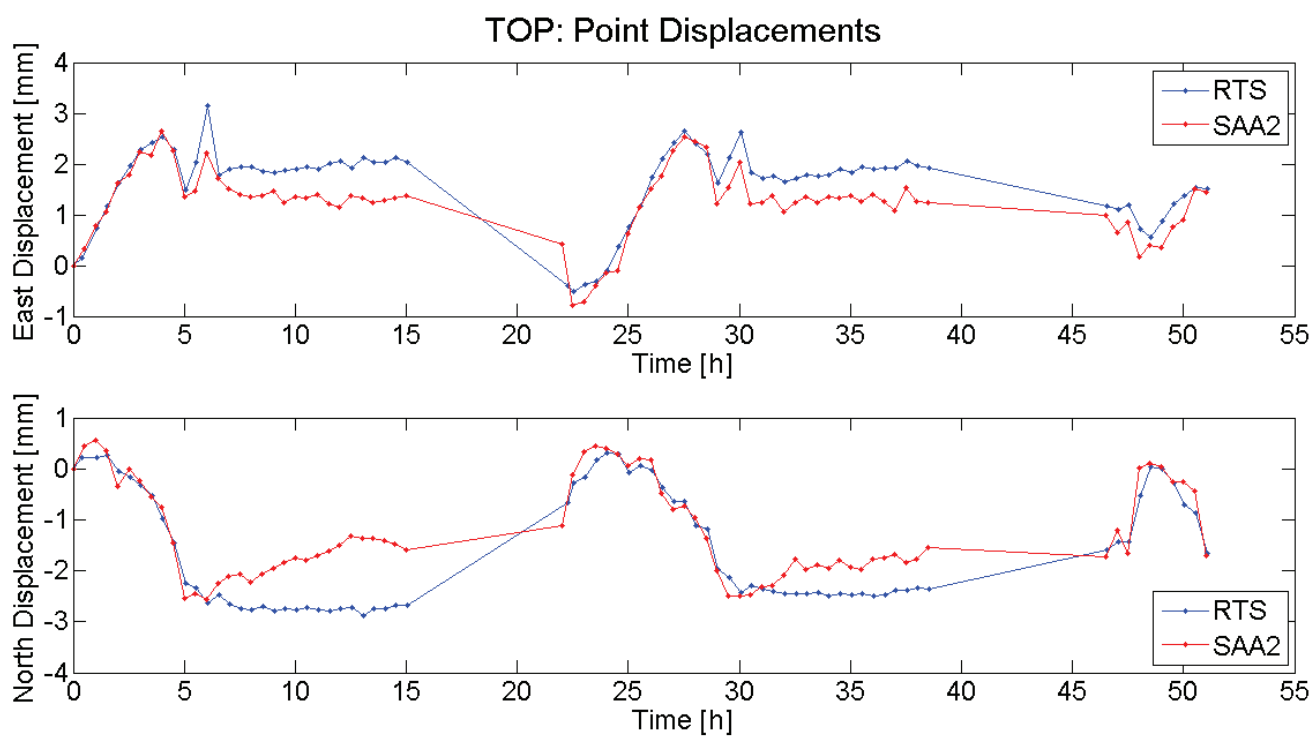

Figure 7: East and North displacements for SAA2 (red) and RTS (blue) for the TOP point.

Figure 8 and Figure 9 show the East and North deformations for the MIDDLE point for SAA1 and RTS and SAA2 and RTS respectively. The SAA1 and SAA2 displacements show similar trends as those in Figure 6 and Figure 7, for TOP point, but on a much smaller scale. The top of the pole is free to move, while the bottom is fixed in concrete, so movement tends to increase toward the top.

The TOP data (Figure 6 and Figure 7) indicate better agreement between SAA1 and the RTS, than for SAA2 for some time periods. The SAA2 disagreements correspond to times when the temperature decreases (Hour 5 to Hour 15 and Hour 30 to Hour 38). Similar SAA2-RTS disagreements appear in Figure 5. Close examination of SAA1 data indicate similar discrepancies during the same cooling periods, although greatly attenuated. The MIDDLE data (Figure 8 and 
Figure 9) indicate good agreement for both SAAs. Discrepancy at the TOP, but not the MIDDLE, is thought to be due to the location of the temperature sensor in each SAA, in this case near the MIDDLE of each SAA. Temperature compensation for sensors far from the temperature sensor can suffer if there are spatial temperature gradients. Such gradients could be larger in the cool evening hours. It is also possible that the tube containing SAA2 does not move faithfully with the target during slow cooling.
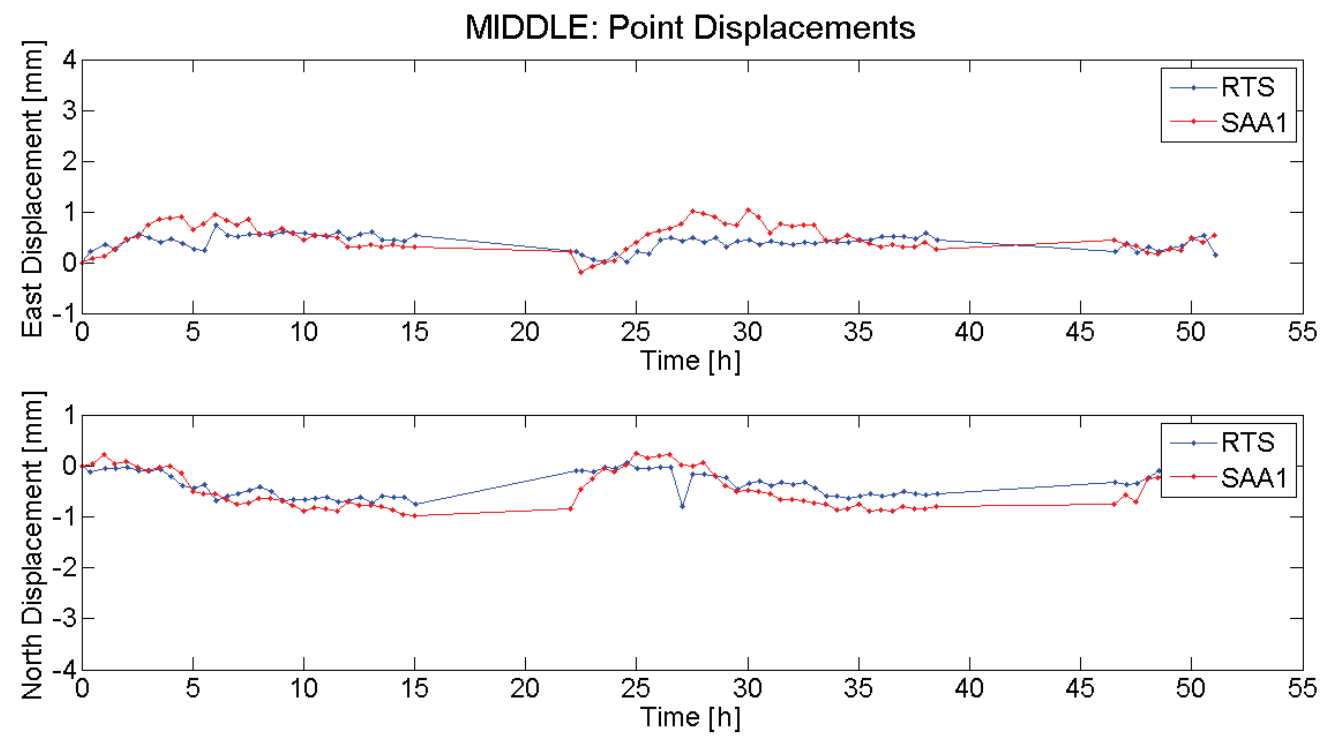

Figure 8: East and North displacements for SAA1 (red) and RTS (blue) for the MIDDLE point.
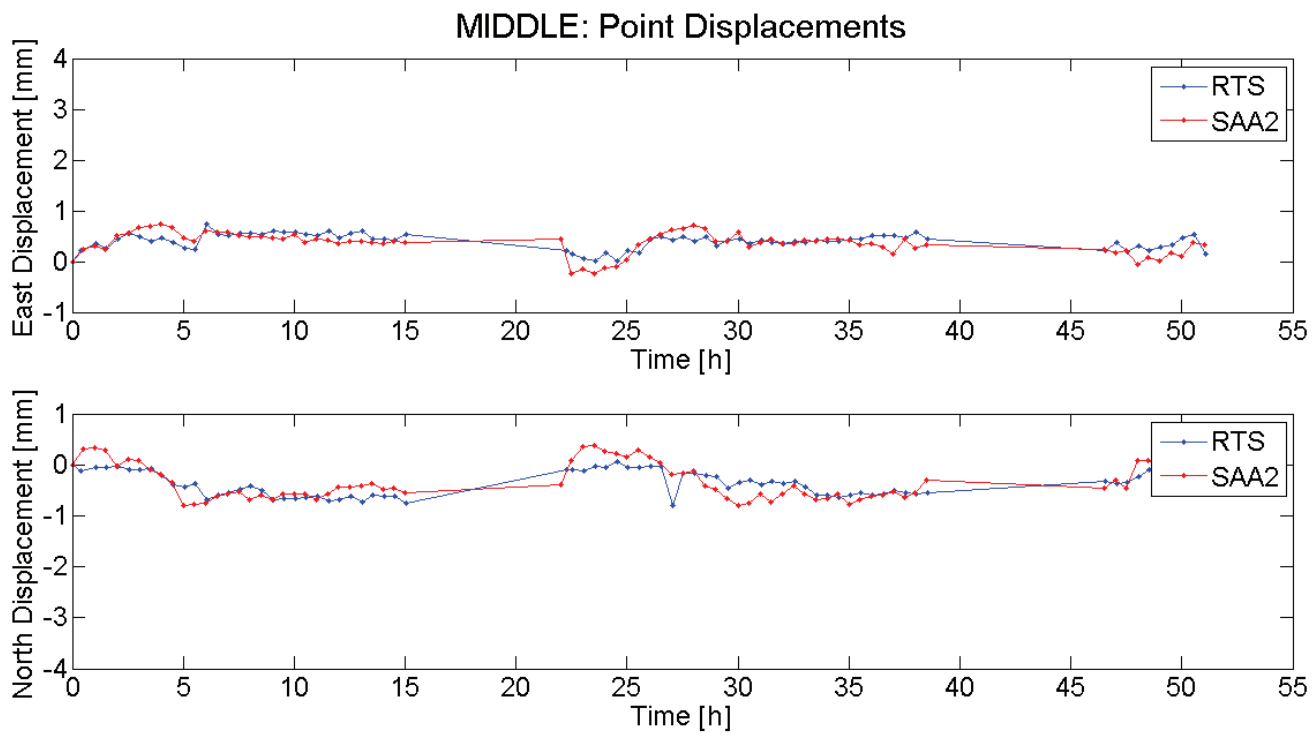

Figure 9: East and North displacements for SAA2 (red) and RTS (blue) for the MIDDLE point.

\section{Summary}

Table 1 shows the root-mean-square (r.m.s.) of the North and East displacement differences between the SAA1 and RTS, SAA2 and RTS for TOP point and MIDDLE point. The r.m.s. of the differences in all cases are less than $1 \mathrm{~mm}$. The average East displacement r.m.s. is $0.30 \mathrm{~mm}$ and the average North displacement r.m.s. is $0.33 \mathrm{~mm}$. 
Table 1: r.m.s. of the East displacement difference and North displacements difference.

\begin{tabular}{|c|c|c|}
\hline Point & $\begin{array}{c}\text { r.m.s. of the East } \\
\text { Displacement Difference }\end{array}$ & $\begin{array}{c}\text { r.m.s. of the North } \\
\text { Displacement Difference }\end{array}$ \\
\hline TOP Point: SAA1 vs. RTS & $0.30 \mathrm{~mm}$ & $0.25 \mathrm{~mm}$ \\
\hline TOP Point: SAA2vs. RTS & $0.49 \mathrm{~mm}$ & $0.60 \mathrm{~mm}$ \\
\hline MIDDLE Point: SAA1 vs. RTS & $0.26 \mathrm{~mm}$ & $0.24 \mathrm{~mm}$ \\
\hline MIDDLE Point: SAA2 vs. RTS & $0.17 \mathrm{~mm}$ & $0.23 \mathrm{~mm}$ \\
\hline
\end{tabular}

\section{Conclusions}

Measurements of the two SAAs agree at the sub-millimetre level. The three-instrument comparison (SAA1, SAA2 and RTS) also demonstrates a sub-millimetre-level agreement between the two SAAs and the RTS. Thus, both objectives (consistency and precision) were achieved over the approximate $20-25{ }^{\circ} \mathrm{C}$ temperature swings of this field test.

\section{Future Work}

Future work will include field testing over other temperature ranges, including higher and belowzero ambients, to further validate the temperature compensation algorithms used with SAAs. More spatial detail will be obtained from the RTS measurements by using more targets, so that closer comparison to the SAA data (SAA provides data at $50 \mathrm{~cm}$ intervals) can be made. Also, a new form of SAA having temperature sensors in every segment will be used, to reduce effects of spatial temperature gradients.

\section{References}

[1] CHRZANOWSKI, A. and A. SZOSTAK-CHRZANOWSKI (2010). "Automation of Deformation Monitoring Techniques and Integration with Prediction Modeling"; Geomatica vol.64, No. 2 pp. 221-231.

[2] DANISCH, L., T. ABDOUN, and M. LOWERY-SIMPSON, (2007). Shape-Acceleration Measurement Device and Method, US Patent 7,296,363.

[3] DANiSCH, L., CHRZANOWSKI, A., BOND, J., and BAZANOWSKI, M. (2008). "Fusion of geodetic and MEMS sensors for integrated monitoring and analysis of deformations," presented at 13th FIG International Symposium on Deformation Measurements and Analysis, Lisbon, Portugal, May 12-15, 2008.

[4] DANISCH, L., T. PATTERSON, and J. FLETCHER (2011). "MEMS-Array Monitoring of a Dam", in Proceedings of Canadian Dam Association Annual Conference. Fredericton, NB. October 15-20, 2011.

[5] DUNNICLIFF, J., (1993). Geotechnical Instrumentation for Monitoring Field Performance, John Wiley and Sons, New York. 